\title{
The effectiveness and side effects of conformal external beam radiotherapy combined with high-dose-rate brachytherapy boost compared to conformal external beam radiotherapy alone in patients with prostate cancer
}

\author{
Beata Smolska-Ciszewska*, Leszek Miszczyk, Brygida Białas, Marek Fijałkowski, Grzegorz Plewicki, \\ Marzena Gawkowska-Suwińska, Monika Giglok, Katarzyna Behrendt, Elżbieta Nowicka, Aleksander Zajusz \\ and Rafał Suwiński
}

\begin{abstract}
Background: Clinical data that compare external-beam radiotherapy (EBRT) combined with high-dose-rate brachytherapy (HDR-BT) boost versus EBRT alone are scarce. The analysis of published studies suggest that biochemical relapse-free survival in combined EBRT and HDR-BT may be superior compared to EBRT alone. We retrospectively examined the effectiveness and tolerance of both schemes in a single center study.

Methods: Between March 2003 and December 2004, 229 patients were treated for localized T1-T2NOM0 prostate cancer. Median age was 66 years (range, 49 - 83 years). PSA level ranged from 0.34 to $64 \mathrm{ng} / \mathrm{ml}$ (median 12.3 ng/ml) and Gleason score ranged from 2 to 10. The analysis included 99 patients who underwent EBRT with HDR-BT (group A) and 130 patients who were treated with EBRT alone (group B).

Results: Median follow-up was 6 years. Biochemical relapses occurred in 34\% vs. 22\% ( $p=0.002$ ), local recurrences in 17\% vs. $5 \%(p=0.002)$, and distant metastases in $11 \%$ vs. $6 \%(p=0.179)$ of patients in groups $A$ and $B$, respectively. Five-year biochemical relapse-free survival was $67 \%$ vs. $81 \%(p=0.005)$, local recurrence-free survival $95 \%$ vs. $99 \%(p=0.002)$, metastases-free survival $95 \%$ vs. $94 \%(p=0.302)$ for groups $A$ and $B$, respectively. Five-year overall survival was $85 \%$ in both groups $(p=0.596)$. Grade $2 / 3$ late $\mathrm{Gl}$ complications appeared in $9.2 \%$ and $24.8 \%(p=0.003)$, respectively. Grade $2 / 3$ late $\mathrm{GU}$ symptoms occurred in $12 \%$ in both groups.

Conclusions: Although because of the retrospective character of the study and nonrandomized selection of fractionation schedule the present conclusions had limitations EBRT alone appeared more effective than EBRT combined with HDR-BT. It was likely the result of the less frequent use of androgen deprivation therapy (ADT) for combined scheme group, too low dose in a single BT fraction or inadequate assumptions regarding fractionation sensitivity of prostate cancer.
\end{abstract}

Keywords: Brachytherapy, High-dose-rate, Prostate cancer, Radiotherapy

\footnotetext{
*Correspondence: gorbea@poczta.onet.pl

Maria Skłodowska-Curie Memorial Cancer Center and Institute of Oncology,

Gliwice Branch, UI. Wybrzeże Armii Krajowej 15, Gliwice 44-100, Poland
} 


\section{Background}

Radical prostatectomy (RP) and radiotherapy are two basic methods of curative treatment for prostate cancer (PC). Radiation treatment can be conducted using external beam radiotherapy (EBRT) alone, interstitial brachytherapy (BT) alone or may combine both of these methods. Comparison of different treatment methods for early stage prostate cancer: low-dose-rate BT (LDR-BT), EBRT <72 Gy, EBRT >72 Gy, combined LDR-BT and RP indicated that biochemical relapse-free survival (bRFS) was inferior in low-dose EBRT, compared to the other groups in all prognostic subsets and was not improved by use of ADT [1]. A systematic review of treatment methods for PC by Grimm et al. revealed that the decision on treatment method may depend on risk group. In low-risk disease BT alone might be the choice, for intermediaterisk disease the combination of EBRT and BT appears equivalent to BT alone, for high-risk patients combined therapies that incorporate EBRT and BT plus or minus androgen deprivation therapy (ADT) appear favorable [2].

Most of the published reports suggest that $\alpha / \beta$ ratio for PC is low, PC may be sensitive, thus, to high fractional doses $[3,4]$. Hypofractionation enables the application of higher single fractions in a shorter overall treatment time. This method is investigational and may be used for EBRT, fractionated stereotactic body radiotherapy and BT [5-8].

Brachytherapy is one of hypofractionated radiotherapy methods. It can not only shorten the overall treatment time and limit the geographical miss, but also enables escalation of the total dose in the target and reduce the dose in surrounding normal tissues. Permanent or temporary implants are used alone in low-risk (LR) PC patients or are combined with EBRT in intermediate (IR) or high-risk (HR) groups [9]. Clinical data that compare toxicity and efficacy of EBRT combined with HDR-BT boost and EBRT alone are scarce [10-16].

A systematic review of radiotherapy for PC by Pieters et al. revealed that the combination of EBRT and HDR$\mathrm{BT}$ resulted in a better bRFS and OS compared to highdose EBRT or EBRT combined with LDR-BT [17].

In this report we retrospectively evaluate the effectiveness and normal tissue reactions of EBRT combined with HDR-BT boost in comparison to EBRT alone for PC. While due to nonrandomized selection of fractionation schedules such study has apparent limitations it may provide an interesting insight of the clinical outcomes that can be confronted with the outcomes of other published series.

\section{Methods}

\section{Patients' characteristics}

Between March 2003 and December 2004, 229 patients with T1-T2N0M0 PC were treated. The analysis included 99 patients who underwent EBRT with HDR-BT (group A) and 130 patients treated with EBRT alone to the median dose of 74 Gy (group B). The median age of the group was 66 years (range, 49 - 83 years). Patients treated with EBRT alone were slightly older than those treated with the combined scheme (67 vs. $65, \mathrm{p}=0.046$ Mann-Whitney U test). All patients had biopsy-proven adenocarcinoma of the prostate, Gleason score ranged from 2 to 10. Pretreatment prostate-specific antigen (PSA) was known in all cases and ranged from 1.7 to $64 \mathrm{ng} / \mathrm{ml}$ (median $12.3 \mathrm{ng} / \mathrm{ml}$ ). Prior to initiation of treatment, all patients underwent a complete clinical examination and laboratory tests (blood count, liver and renal function). Staging procedures included digital rectal examination (DRE), transrectal ultrasound (TRUS) of the prostate or pelvic magnetic resonance imaging. Distant metastases were excluded by abdomen and pelvis computed tomography $(\mathrm{CT})$ or ultrasound examination, chest X-ray and bone scan. The clinical stage was defined according to 2009 TNM classification system [18]. Patients were divided into risk groups according to D'Amico [19]. The clinical characteristics of the groups are summarized in Table 1. In general, the groups were comparable with respect to major prognostic factors, except for use of adjuvant ADT (further explained below).

The combined treatment was considered in men with good performance status and with prostate suitable for TRUS-guided implantation. Patients with a history of transurethral resection or with PC in clinical T3 stage according to the brachytherapy protocol were excluded. The decision on treatment method - EBRT alone or combined scheme was made by patients. They were informed about treatment methods, all possible complications, the advantages and disadvantages of the proposed schedules and were given liberty to choose a preferred therapy.

\section{Androgen deprivation therapy}

Neoadjuvant ADT was used in $90 \%$ patients in both groups. Adjuvant ADT was applied in 97 men: 32/99 (32\%) in group A and 65/130 (50\%) in group B ( $p=0.008$ Mann-Whitney $\mathrm{U}$ test). Median duration of ADT in group A was 5.9 months compared to 12.9 months in group $B(p<0.001$ MannWhitney $U$ test). The characteristics of ADT is summarized in Table 1.

\section{External beam radiotherapy}

All patients underwent EBRT with 6-20 MV photons generated by a linear accelerator. Three-dimensional conformal planning technique was based on CT in all cases. Patients were placed in a supine position. Precise and reproducible patient immobilization was achieved using thermoplastic mask system (Orfit Industries, Belgium). Clinical target volume (CTV) included the prostate and base of seminal vesicles, and pelvic lymph nodes in most of HR patients. Planning target volume (PTV) expansion of 10-15 mm was 
Table 1 Characteristics of 229 patients treated with EBRT-BT and EBRT alone for prostate cancer

\begin{tabular}{|c|c|c|c|c|}
\hline Characteristics & $\begin{array}{l}\text { Total } \\
(\mathrm{N}=229)\end{array}$ & $\begin{array}{l}\text { Group A EBRT-BT } \\
(\mathrm{N}=99)\end{array}$ & $\begin{array}{l}\text { Group B EBRT } \\
(\mathrm{N}=130)\end{array}$ & p (Mann-Whitney U test \\
\hline Age [years] & & & & 0.046 \\
\hline Median & 66 & 65 & 67 & \\
\hline Range & $49-83$ & $49-83$ & $51-80$ & \\
\hline Zubrod score & & & & 0.1 \\
\hline 0 & $196(85.6 \%)$ & 89 (89.9\%) & 107 (82.3\%) & \\
\hline 1 & $32(14 \%)$ & $10(10.1 \%)$ & $22(16.9 \%)$ & \\
\hline 2 & $1(0.4 \%)$ & $0(0 \%)$ & $1(0.8 \%)$ & \\
\hline$P S A[n g / m l]$ & & & & 0.463 \\
\hline Mean & 16.3 & 15.4 & 16.9 & \\
\hline Median & 12.3 & 11.8 & 13.9 & \\
\hline Range & $1.7-64$ & $3.9-56.6$ & $1.7-64$ & \\
\hline$P S A[n g / m l]$ & & & & 0.962 \\
\hline$<10$ & $81(35.4 \%)$ & $33(33.3 \%)$ & 48 (36.9\%) & \\
\hline $10-20$ & 91 (39.7\%) & $44(44.4 \%)$ & 47 (36.2\%) & \\
\hline$>20$ & $57(24.9 \%)$ & $22(22.2 \%)$ & $35(26.9 \%)$ & \\
\hline Gleason score & & & & 0.122 \\
\hline $2-6$ & $115(67.7 \%)$ & $67(67.7 \%)$ & $88(67.7 \%)$ & \\
\hline 7 & 39 (17\%) & $18(18.2 \%)$ & $21(16.2 \%)$ & \\
\hline $8-10$ & $22(9.6 \%)$ & $8(8.1 \%)$ & $14(10.8 \%)$ & \\
\hline unknown & $13(5.6 \%)$ & $6(6 \%)$ & $7(5.3 \%)$ & \\
\hline Clinical T stage & & & & 0.118 \\
\hline T1b & $8(3.5 \%)$ & $2(2 \%)$ & $6(4.6 \%)$ & \\
\hline T1c & $95(41.7 \%)$ & 39 (39.4\%) & $56(43.1 \%)$ & \\
\hline T2a & $49(21.3 \%)$ & $19(19.2 \%)$ & $30(23.1 \%)$ & \\
\hline $\mathrm{T} 2 \mathrm{~b}$ & $26(11.3 \%)$ & $12(12.1 \%)$ & $14(10.7 \%)$ & \\
\hline $\mathrm{T} 2 \mathrm{c}$ & $51(22.2 \%)$ & $27(27.3 \%)$ & $24(18.5 \%)$ & \\
\hline Risk group & & & & 0.310 \\
\hline LR & $48(21 \%)$ & $18(18.2 \%)$ & $30(23.1 \%)$ & \\
\hline IR & $82(35.8 \%)$ & $35(35.3 \%)$ & $47(36.1 \%)$ & \\
\hline HR & $99(43.2 \%)$ & $46(46.5 \%)$ & $53(40.8 \%)$ & \\
\hline \multicolumn{5}{|l|}{$A D T$} \\
\hline Neoadjuvant & $206(90 \%)$ & $88(89.8 \%)$ & 118 (90.7\%) & 0.639 \\
\hline Adjuvant & $97(42.4 \%)$ & $32(32.3 \%)$ & $65(50 \%)$ & 0.008 \\
\hline Salvage & $63(27.4 \%)$ & $37(37.4 \%)$ & $26(20 \%)$ & 0.004 \\
\hline no & $11(4.8 \%)$ & $4(4 \%)$ & $7(5.3 \%)$ & \\
\hline Total duration of $A D T$ [months] & & & & $<0.001$ \\
\hline Median & 98.9 & 6 & 13 & \\
\hline Range & $0.5-79$ & $0.7-55.6$ & $0.5-79$ & \\
\hline Whole pelvis EBRT & $84(36.7 \%)$ & $40(40.4 \%)$ & $44(33.9 \%)$ & 0.309 \\
\hline PSA nadir $[\mathrm{ng} / \mathrm{ml}]$ & & & & 0.049 \\
\hline Median & 0.04 & 0.07 & 0.03 & \\
\hline
\end{tabular}


Table 1 Characteristics of 229 patients treated with EBRT-BT and EBRT alone for prostate cancer (Continued)

\begin{tabular}{|c|c|c|c|c|}
\hline Range & $0-12.3$ & $0-12.3$ & $0-3.1$ & \\
\hline Time to obtain PSA nadir [months] & & & & 0.007 \\
\hline Median & 6.3 & 5.3 & 8.7 & \\
\hline Range & $0.9-74.9$ & $1.1-58.4$ & $0.9-74.9$ & \\
\hline
\end{tabular}

applied, except for the posterior expansion of 7-8 $\mathrm{mm}$. Organs at risk were rectum, urinary bladder, femoral heads and bowel.

Radiation dose was prescribed and specified in the reference point according to the guidelines of the International Commission on Radiation Units Report 50 and 62. Treatment plans were developed with Eclipse treatment-planning system (Varian Medical System, Palo Alto, CA, USA). Varian Clinac linear accelerators (Varian Medical System, Palo Alto, CA, USA) were used in radiation treatment. Portal vision and in vivo dosimetry were used to control the referral points of the dosimetric parameters. The final decision on whole pelvis irradiation depended on risk factors and oncologist's decision (40/99 (40.4\%) in group A and 44/130 (33.9\%) in group B received pelvic RT). Median external beam dose to the pelvis was 44 Gy (range, 44-50 Gy) in 22 fractions (range, 22-25 fractions). Median dose to the prostate was 74 Gy (range, 70-76 Gy) in 37 fractions (range, 35-39 fractions) in group B and 54 Gy (range, 51-56 Gy) in 27 fractions (range, 27-29 fractions) in group A. The total duration of radiation treatment in group A was shorter : 34-58 days (median 41) vs. 41-79 days (median 52) in groups A and $\mathrm{B}$, respectively.

\section{Brachytherapy}

Patients were placed in dorsal lithotomic position under spinal or general anesthesia. A Foley catheter was used to fill the urinary bladder with $150 \mathrm{ml}$ of sterile water. A biplaner TRUS was performed to view the prostate, seminal vesicles, urethra, bladder and rectum and prepare a preplan. TRUS probe was fixed to the stepper. The entire prostate gland was defined as clinical target volume (CTV) for HDR-BT. Treatment planning using PLATO Complete (Nucletron, Veenendaal, The Netherlands) was performed with the following constraints: maximum dose to urethra and rectum had to be less than $120 \%$ and $7 \mathrm{~Gy}$, respectively. Needles (from nine to eighteen) were implanted under the TRUS guidance. The location of each needle was visualized and the implant was reconstructed by the treatment planning system. A single fraction of $10 \mathrm{~Gy}$ was applied by a HDR 192-Ir stepping source unit (MicroSelectron $^{\mathrm{Tm}}$, Nucletron, Veenendaal, The Netherlands). The dose was calculated on the prostate capsule. A single-fraction HDR-BT boost was given before, during or after EBRT. The date of the procedure depended on patient performance status or waiting time in Brachytherapy Department.

\section{Follow-up and statistical analysis}

Median follow-up time was 6 years (range 0.5-7.6). It was 5.9 years (range, $0.6-7.7$ years) and 6 years (range, 1-7.6 years) for EBRT alone and EBRT-BT, respectively. Patients were evaluated every 3 months during the first year and every 6 months thereafter. Each evaluation included a clinical examination, DRE and PSA concentration. Acute and late radiation toxicity was evaluated according to the European Organization for Research and Treatment of Cancer / Radiation Therapy Oncology Group (EORTC/RTOG) scoring system [20].

Biochemical failure after RT was defined according to the Radiation Therapy Oncology Group (RTOG) - American Society of Therapeutic Radiology and Oncology (ASTRO) Phoenix consensus criteria: PSA nadir +2 ng/ml [21]. Local recurrence was defined as local progression detected by a biopsy. The biopsy was performed in patients with biochemical relapse and no evidence of distant metastases. Metastases-free survival (MFS) reflected all distant failures. Overall survival (OS) reflected all deaths, both cancer related or not. RFS and bRFS were calculated from the end of the treatment to the time when any recurrence was detected or to the last follow-up visit. MFS and OS were calculated from the beginning of the treatment to a distant failure, death or the last follow-up visit. The actuarial rates of bRFS, RFS, MFS and OS were estimated according to the Kaplan-Meier method. The statistical significance of the differences between actuarial curves was calculated using the log-rank test. The Cox proportional hazard regression analysis was applied in the multivariable models. Student $\mathrm{t}$ or Mann-Whitney $\mathrm{U}$ tests were applied to test of the hypothesis that two populations are the same, against an alternative hypothesis. The difference was considered significant if the $\mathrm{p}$ value was less than 0.05 .

\section{Results}

\section{PSA nadir}

The median nadir value of PSA was lower in group B compared to group A (0.03 vs. $0.07 \mathrm{ng} / \mathrm{ml}, \mathrm{p}=0.049$ Mann-Whitney U test). The time to obtain PSA nadir in the group B was significantly longer (8.7 vs. 5.2 months, $\mathrm{p}=0.007$ Mann-Whitney U test).

\section{Treatment outcome}

At the median follow-up of 6 years, 56/229 (24\%) biochemical relapses, 23/229 (10\%) local recurrences and 19/ 229 (8\%) distant metastases occurred in both groups. 
Biochemical recurrences were significantly more frequent in group A (34/99 (34\%) vs. $22 / 130(17 \%)$ in group B, p = 0.002 Mann-Whitney U test). Local recurrences also occurred more often in group A (17/99 (17\%) vs. 6/130 (5\%), $\mathrm{p}=0.002 \mathrm{Mann}-$ Whitney U test). Distant metastases appeared in 11/99 (11\%) patients in group A and in 8/130 (6\%) in group B ( $\mathrm{p}=0.179$, Mann-Whitney $\mathrm{U}$ test). The most common sites of metastases were bones (73\%), lymph nodes (31\%), lungs (16\%) and the liver (5\%).

To date, death occurred in 44/229 (19\%) patients in both groups: 17/99 (17\%) died in group A and 27/130 (21\%) in group B $(\mathrm{p}=0.495$, Mann-Whitney $U$ test). Ten patients (4\%) died of prostate cancer: $5 / 99(5 \%)$ in group A and $5 / 130(4 \%)$ in group B $(p=0.659$, MannWhitney U test). The remaining 34 patients (15\%) died due to other causes.

The 5 -year bRFS rate for group B was $81 \%$ and $67 \%$ for group A ( $\mathrm{p}=0.005, \log$-rank test) (Figure 1$)$. In the univariable analysis treatment scheme $(\mathrm{p}=0.005)$ and risk group $(p=0.041)$ were significant predictors of the risk of biochemical failure (Table 2). In the multivariable Cox proportional hazard regression analysis the treatment scheme $(\mathrm{p}=0.016)$ appeared as an independent predictor of the risk of biochemical relapse.

The 5-year RFS rate was $99 \%$ for group B and $95 \%$ for group A ( $\mathrm{p}=0.002$, log-rank test) (Figure 2). In the univariable analysis treatment schedule $(\mathrm{p}=0.002)$ was found to be associated with local failure (Table 2). The multivariable Cox proportional hazard regression analysis also showed that treatment schedule $(\mathrm{p}=0.045)$ was associated with higher RFS.

Five-year MFS was $95 \%$ and $94 \%$ for groups A and B, respectively $(\mathrm{p}=0.302$, log-rank test). Five-year OS was $85 \%$ in both groups $(\mathrm{p}=0.596, \log$-rank test). The Cox proportional hazard regression analysis revealed that risk group ( $\mathrm{p}=0.049$ ) was significant for prediction of MFS and patient's age $(\mathrm{p}=0.038)$ was significant for prediction of OS. We also estimated the survival within each risk group. The results are summarized in Table 3.

\section{Side effects}

Grade 2 and 3 acute gastrointestinal (GI) symptoms occurred in 18/99 (18.2\%) in group A and in 36/130 (27.7\%) in group $B(p=0.094$, Mann-Whitney $U$ test). Grade 2 and 3 acute genitourinary $(\mathrm{GU})$ reactions were observed in $42 / 99$ (42.4\%) and in 43/130 (33.1\%) ( $\mathrm{p}=0.148$, Mann-Whitney U test), respectively. None of the patients developed grade 4 GI or GU early complications. The most frequent acute reactions during radiotherapy were diarrhea of low or moderate severity and polyuria. Six patients required treatment interruption because of severe diarrhea and paralytic subileus.

Grade 2 late GI side effects appeared in 9/98 (9.2\%) cases in group A and grade 2 and 3 late GI complications in $31 / 125(24.8 \%)$ patients in group B $(\mathrm{p}=0.003$, MannWhitney $U$ test). No patient developed grade 4 GI late symptoms. The most frequent late reactions were chronic diarrhea and intermittent rectal bleedings. Four patients required the treatment of argon plasma laser coagulation in group B. Grade 2 and 3 late GU symptoms occurred in $12 \%$ in both groups (12/98 in group A and 15/125 in group B). Urethral strictures and hemorrhagic cystitis caused a need for catheterization, required endourethral incisions, transurethral resection of the prostate or electrocoagulation of teleangiectasis, which appeared in 3/98 (3.1\%) in group A and in 5/125 (4\%) in group B. No patient developed grade $4 \mathrm{GU}$ late complications. The median time interval for occurrence of late GI and GU

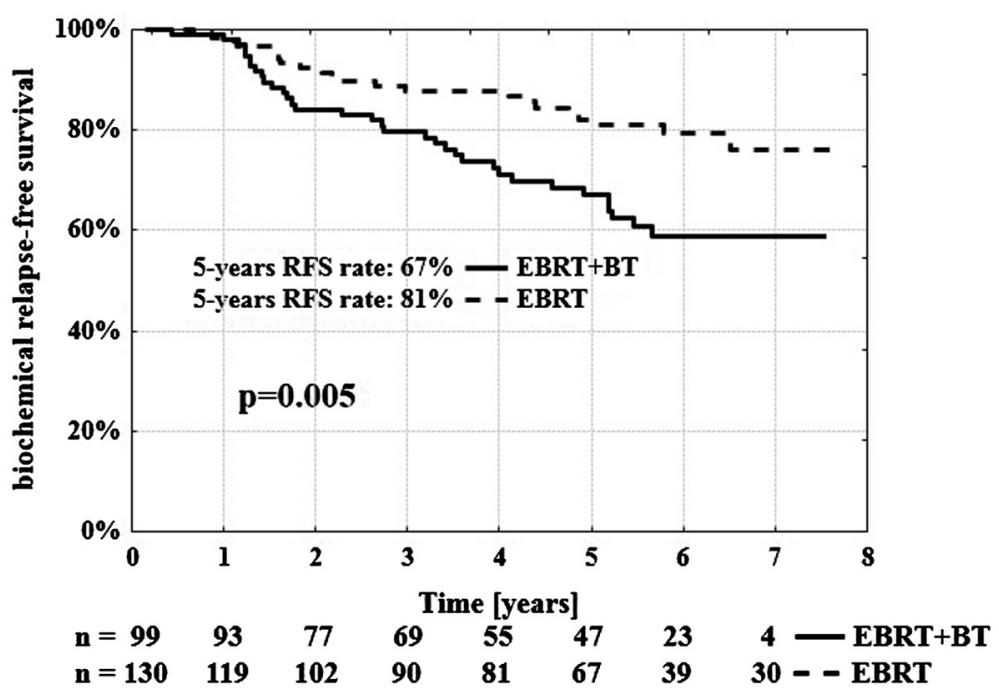

Figure 1 Biochemical relapse-free survival rate according to treatment schedule. n-number of patients at risk. 
Table 2 Variables influencing actuarial rates of bRFS, RFS, MFS, OS

\begin{tabular}{|c|c|c|c|c|c|c|c|c|}
\hline Characteristic & 5-y bRFS & $\mathrm{p}$ & 5-y RFS & $\mathrm{p}$ & 5-y MFS & $\mathrm{p}$ & $5-y$ OS & $\mathrm{p}$ \\
\hline Treatment scheme & & 0.005 & & 0.002 & & 0.302 & & 0.596 \\
\hline EBRT-BT & 67 & & 95 & & 95 & & 85 & \\
\hline EBRT & 81 & & 99 & & 94 & & 85 & \\
\hline Age-median & & 0.452 & & 0.421 & & 0.948 & & 0.029 \\
\hline$<66$ & 70 & & 95 & & 95 & & 90 & \\
\hline$\geq 66$ & 80 & & 98 & & 94 & & 82 & \\
\hline Risk group & & 0.041 & & 0.894 & & 0.003 & & 0.369 \\
\hline LR & 83 & & 97 & & 98 & & 85 & \\
\hline IR & 81 & & 98 & & 100 & & 88 & \\
\hline HR & 65 & & 96 & & 88 & & 84 & \\
\hline Total duration of ADT [months] & & 0.089 & & 0.748 & & 0.555 & & 0.156 \\
\hline$<9.2$ & 72 & & 100 & & 93 & & 83 & \\
\hline$\geq 9.2$ & 78 & & 96 & & 95 & & 86 & \\
\hline
\end{tabular}

reactions was 22 months. Both GI and GU late side effects occurred earlier in patients treated with EBRT alone.

\section{Discussion}

This study compares two radiotherapy schedules: EBRT combined with HDR-BT and EBRT alone for patients with PC. After median follow-up time of 6 years, significant differences in clinical outcomes were found between the groups.

The literature data provided by Hoskin et al., Guix et al., Zwahlen et al., Kestin et al. [10-14,16] (Table 4) demonstrated better bRFS after EBRT plus HDR-BT, compared to EBRT alone. Combination of both modalities may also improve OS [17]. Such outcome can be explained by high radiation dose that can be prescribed when HDR-BT is combined with EBRT.

The comparison among the studies presented in Table 4 is difficult due to the apparent differences in the applied dose of EBRT and HDR-BT schemes, diverse definitions of risk groups or biochemical failure. However, we noted that the total dose applied in EBRT alone schedule [10,11,14-16] was lower (66-70 Gy) than recommended by the current guidelines. The adequate dose of 76 Gy in EBRT alone group was applied only in the study by Guix et al. $[12,13]$ (Table 4).

While because of the retrospective character of the study and nonrandomized selection of fractionation schedule the present results have apparent limitations, they suggest relatively low effectiveness of the combined scheme.

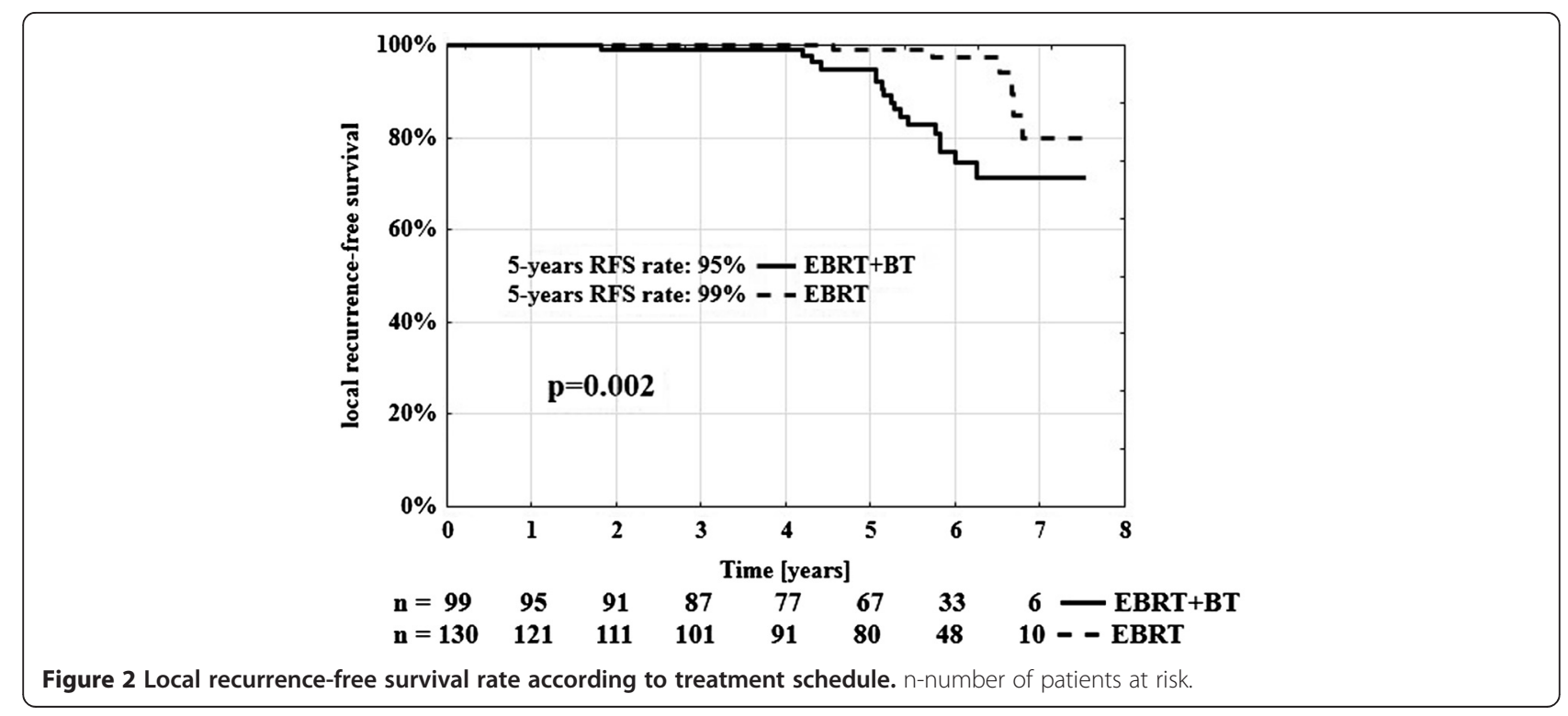


Table 3 Five-year survivals according to risk groups and treatment methods

\begin{tabular}{|c|c|c|c|c|c|c|c|}
\hline & & $\begin{array}{l}\text { LR group } \\
(\mathrm{N}=48)\end{array}$ & $p$ & $\begin{array}{l}\text { IR group } \\
(\mathrm{N}=82)\end{array}$ & $p$ & $\begin{array}{l}\text { HR group } \\
(\mathrm{N}=99)\end{array}$ & $p$ \\
\hline \multirow[t]{2}{*}{ 5-y bRFS } & EBRT-BT & $77 \%$ & 0.16 & $73 \%$ & 0.06 & $59 \%$ & 0.1 \\
\hline & EBRT & $88 \%$ & & $88 \%$ & & $71 \%$ & \\
\hline \multirow[t]{2}{*}{ 5-y RFS } & EBRT-BT & $94 \%$ & 0.07 & $96 \%$ & 0.03 & $94 \%$ & 0.13 \\
\hline & EBRT & $100 \%$ & & $100 \%$ & & $100 \%$ & \\
\hline \multirow[t]{2}{*}{ 5-y MFS } & EBRT-BT & $100 \%$ & 0.42 & $100 \%$ & 0.15 & $88 \%$ & 0.98 \\
\hline & EBRT & $96 \%$ & & $100 \%$ & & $87 \%$ & \\
\hline \multirow[t]{2}{*}{$5-y$ OS } & EBRT-BT & $89 \%$ & 0.31 & $88 \%$ & 0.66 & $82 \%$ & 0.72 \\
\hline & EBRT & $83 \%$ & & $87 \%$ & & $85 \%$ & \\
\hline
\end{tabular}

This finding is inconsistent to previous reports demonstrating the superiority of the combined therapy. Unexpectedly, in spite of initial assumptions based on radiobiological premises i.e.: the prostate tumor's $\alpha / \beta$ ratio of 1.5 Gy [22], the scheme was not even as effective as EBRT alone. One may postulate that the assumed $\alpha / \beta$ ratio value for prostate cancer is not appropriate since some studies suggest is higher value [23-26], including the studies in which HDR brachytherapy was used [27].

Notably, Roberts et al. [27] compared bRFS results for PC patients who received combined EBRT and HDR-BT with expected projections that were based on dose/fractionation/response parameter values derived from EBRT alone. Tumor control rates after EBRT and HDR-BT, were lower in many cases then predicted from linearquadratic (LQ) parameter estimates from EBRT alone. Lower than expected control rates were associated with BT doses higher than 30 Gy, 1-2 high-dose fractions, 9 fractions (BT alone), doses per fraction of 9-15 Gy, and treatment in only 1 week. It could be explained by cold spots in brachytherapy dose distribution or uncertain applicability of the LQ model at high doses per fraction [27]. This study supports our findings, that a combined scheme of one high-dose BT fraction of 10 Gy may provide biochemical and local control lower than expected.

Relatively small total dose in combined BT and EBRT used in the present study may also contribute to the unsatisfactory local effectiveness. In some studies BT was administered in the minimum of 2 fractions that allowed to escalate the dose, limit the toxicity and avoid the risk of nonhomogenous dose distribution (Table 4). On the other hand, escalation of EBRT dose with one BT fraction may appear effective, as shown by Agoston et al. [28] or Boladeras et al. [29].

Another factor contributing to the observed outcome could be the difference in use of hormone treatment in the analyzed groups: adjuvant ADT was more often applied in the EBRT alone than in the group treated with the combined method ( $50 \%$ vs. $32 \%, \mathrm{p}=0.008$ ). The total time of the applied ADT was also longer in the EBRT alone (12.9 vs. 5.9 months, $\mathrm{p}<0.001)$. In Agoston et al. and Boladeras et al. studies that show a favorable

Table 4 Selected published trials on EBRT with HDR-BT in prostate cancer

\begin{tabular}{|c|c|c|c|c|c|c|c|}
\hline \multirow{3}{*}{$\begin{array}{l}\text { Study } \\
\text { Hoskin 2007/2012 } \\
{[10,11] \text { (7-year survival) }}\end{array}$} & \multirow{2}{*}{$\begin{array}{l}\text { scheme } \\
\text { EBRT -BT }\end{array}$} & dose & $E D_{2} \alpha / \beta=1.5 \mathrm{~Gy}$ & \multirow{2}{*}{$\frac{\mathrm{EQD}_{\mathbf{2}} \boldsymbol{\alpha} / \boldsymbol{\beta}=\mathbf{3} \mathrm{Gy}}{80.21 \mathrm{~Gy}}$} & \multicolumn{2}{|c|}{ 5-year bRFS } & \multirow{2}{*}{$\frac{\text { 5-year OS }}{88 \%(81 \%)}$} \\
\hline & & $35.75 \mathrm{~Gy} / 13 \mathrm{fx}+2 \times 8.5 \mathrm{~Gy}$ & $92 \mathrm{~Gy}$ & & \multicolumn{2}{|c|}{$75 \%(66 \%)$} & \\
\hline & EBRT & $55 G y / 20 f x$ & $66.8 \mathrm{~Gy}$ & 63.25 Gy & \multicolumn{2}{|c|}{$61 \%(48 \%)$} & $89 \%(81 \%)$ \\
\hline \multirow[t]{3}{*}{ Guix 2010/2011 [12,13] } & EBRT -BT & $46 \mathrm{~Gy} / 23 \mathrm{fx}+2 \mathrm{x} 8 \mathrm{~Gy}$ & 89.4 Gy & 81.2 Gy & $\mathbb{R}$ & $\mathrm{HR}$ & - \\
\hline & & & & & $97 \%$ & $96 \%$ & \\
\hline & EBRT & 76 Gy/38fx & 76 Gy & 76 Gy & $90 \%$ & $89 \%$ & - \\
\hline \multirow{2}{*}{$\begin{array}{l}\text { Zwahlen } 2010 \\
\text { [14] (7-year survival) }\end{array}$} & EBRT -BT & 46 Gy/23fx + 3x6Gy or 4x5Gy & 82.1-84.6 Gy & 78-78.4 Gy & \multicolumn{2}{|c|}{$82.5 \%(80.3 \%)$} & $91.9 \%(89.5 \%)$ \\
\hline & EBRT & 70 Gy/35fx & $70 \mathrm{~Gy}$ & 70 Gy & \multicolumn{2}{|c|}{$81.3 \%(71 \%)$} & $88.7 \%(86.2 \%)$ \\
\hline \multirow[t]{2}{*}{ Kestin 2000 [16] } & EBRT + HDR-BT & 46 Gy/23fx + 2-3x5.5-10.5Gy & 79-118 Gy & 74.1-102.7 Gy & \multicolumn{2}{|l|}{$67 \%$} & - \\
\hline & EBRT & $66 \mathrm{~Gy} / 33 \mathrm{fx}$ & $66 \mathrm{~Gy}$ & $66 \mathrm{~Gy}$ & \multicolumn{2}{|l|}{$44 \%$} & - \\
\hline \multirow[t]{2}{*}{ This study (7-year survival) } & EBRT + HDR-BT & $54 G y / 27 f x+1 x 10 G y$ & 86.8 Gy & $80 \mathrm{~Gy}$ & \multicolumn{2}{|c|}{$67 \%(59 \%)$} & $85 \%(80 \%)$ \\
\hline & EBRT & $74 G y / 37 f x$ & 74 Gy & $74 \mathrm{~Gy}$ & \multicolumn{2}{|c|}{$81 \%(76 \%)$} & $85 \%(73 \%)$ \\
\hline
\end{tabular}

$\mathrm{EQD}_{2}$ - equivalent total dose in 2-Gy fractions. 
outcome after single BT boost radiation treatment was accompanied by long adjuvant ADT in most of the patients $[28,29]$.

At last, we note that the bRFS rate in the EBRT alone group in the present study compares favorably to bRFS rates in some published studies on a high-dose radiotherapy $[30,31]$.

The majority of studies on radiotherapy in prostate cancer assessed not only the effectiveness of treatment but also its tolerance. Comparing the toxicity in diverse studies is difficult due to different classifications of radiation reactions or by reporting the incidence of severe side effects only. According to the published studies, acute GU reactions were more intense in the combined scheme groups $[8,14,16]$. By contrast, late GI reactions more often appeared in EBRT alone groups [12,13]. Late GU side effects were comparable in both groups $[10-13,15]$. A similar outcome has been presented in this analysis. In general, the treatment was well tolerated in both groups.

\section{Conclusions}

Although because of the retrospective character of the study and nonrandomized selection of fractionation schedule the present conclusions had limitations, EBRT alone appeared more effective than EBRT combined with HDRBT. It was likely the result of the less frequent use of androgen deprivation therapy in combined scheme group, too low dose in a single BT fraction or inadequate assumptions regarding fractionation sensitivity of prostate cancer. Acute and late GI complications were more severe in the group treated with EBRT alone. Acute GU symptoms were more intense in the EBRT with HDR-BT group, late GU reactions were comparable in both groups.

\section{Abbreviations}

ADT: Androgen deprivation therapy; ASTRO: American Society of Therapeutic

Radiology and Oncology; bRFS: Biochemical relapse-free survival; BT: Brachytherapy; CT: Computed tomography; CTV: Clinical target volume; DRE: Digital rectal examination; EBRT: External beam radiotherapy; EORTC: European Organization for Research and Treatment of Cancer; $\mathrm{EQD}_{2}$ : Equivalent total dose in 2-Gy fractions; Fx: Fraction; Gy: Gray; HDR-BT: High-dose-rate brachytherapy boost; HR: High-risk; IR: Intermediate-risk; LDR: Low-dose-rate; LQ: Linear-quadratic; LR: Low-risk; MFS: Metastases-free survival; OS: Overall sunvival; PC: Prostate cancer; PSA: Prostate specific antigen; PTV: Planning target volume; RFS: Recurrence-free survival; RP: Radical prostatectomy; RTOG: Radiation Therapy Oncology Group; SBRT: Stereotactic body radiotherapy; TRUS: Transrectal ultrasound.

\section{Competing interests}

The authors declare that they have no competing interests.

\section{Authors' contributions}

BSC participated in design of the study, collected clinical data, prepared statistical analysis, interpreted the data and prepared draft of the manuscript. LM participated in design of the study, was involved in revision of the manuscript and interpretation of the data. BB was involved in revision of the manuscript. MF helped to collect clinical data. GP helped to collect clinical data. MGS was involved in revision of the manuscript. MG helped to collect clinical data. KB helped in statistical analysis. EN helped to collect clinical data. AZ was involved in revision of the manuscript. RS participated in study design, was involved in drafting of the manuscript and its revision. All authors read and approved the final manuscript.
Received: 20 November 2014 Accepted: 20 February 2015

Published online: 07 March 2015

\section{References}

1. Kupelian PA, Potters L, Khuntia D, Ciezki JP, Reddy CA, Reuther AM, et al. Radical prostatectomy, external beam radiotherapy $<72 \mathrm{~Gy}$, external beam radiotherapy $\geq 72 \mathrm{~Gy}$, permanent seed implantation, or combined seeds/ external beam radiotherapy for stage T1-T2 prostate cancer. Int J Radiat Oncol Biol Phys. 2004;58:25-33.

2. Grimm P, Billiet I, Bostwick D, Dicker AP, Frank S, Immerzeel J, et al. Comparative analysis of prostate-specific antigen free survival outcomes for patients with low, intermediate and high risk prostate cancer treatment by radical therapy. Results from the Prostate Cancer Results Study Group. BJU Int. 2012;109 Suppl 1:22-9.

3. Miralbell R, Roberts SA, Zubizarreta E, Hendry JH. Dose-fractionation sensitivity of prostate cancer deduced from radiotherapy outcomes of 5,969 patients in seven international institutional datasets: $\alpha / \beta=1.4(0.9-2.2)$ Gy. Int J Radiat Oncol Biol Phys. 2012;82:e17-24.

4. Dasu A. Is the alpha/beta value for prostate tumours low enough to be safely used in clinical trials? Clin Oncol. 2007;19:289-301.

5. Pollack A, Walker G, Buyyounouski M, Horwitz E, Price R, Feigenberg S, et al. Five year results of a randomized external beam radiotherapy hypofractionation trial for prostate cancer. Int J Radiat Oncol Biol Phys. 2011:81 Suppl 1:S1.

6. Kuban DA, Noguaras-Gonzalez GM, Hamblin L, Lee AK, Choi S, Frank SJ, et al. Preliminaary report of a randomized dose escalation trial for prostate cancer using hypofractionation. Int J Radiat Oncol Biol Phys. 2010;78 Suppl 1:S58-9.

7. King CR, Freeman D, Kaplan I, Fuller D, Bolzicco G, Collins S, et al. Stereotactic body radiotherapy for localized prostate cancer: pooled analysis from a multi-institutional consortium of prospective phase II trials. Radiat Oncol. 2013;109:217-21.

8. Prada PJ, Gonzales H, Fernandez J, Jiménez I, Iglesias A, Romo I. Biochemical outcome after high-dose-rate intensity modulated brachytherapy with external beam radiotherapy: 12 years of experience. BJU Int. 2011;7:1-7.

9. Hoskin PJ, Colombo A, Henry A, Niehoff P, Paulsen Hellebust T, Siebert FA, et al. GEC/ESTRO recommendations on high dose rate afterloading brachytherapy for localised prostate cancer: an update. Radiother Oncol. 2013;107:325-32.

10. Hoskin PJ, Motohashi K, Bownes P, Bryant L, Ostler P. High dose rate brachytherapy in combination with external beam radiotherapy in the radical treatment of prostate cancer: initial results of a randomised phase three trial. Radiother Oncol. 2007;84:114-20.

11. Hoskin PJ, Rojas AM, Bownes PJ, Lowe GJ, Ostler PJ, Bryant L. Randomised trial of external beam radiotherapy alone Or combined with high-dose-rate brachytherapy boost for localised prostate cancer. Radiother Oncol. 2012;103:217-22.

12. Guix B, Bartina J, Tello J, Henriquez I, Quinzaños L, Lacorte T, et al. Dose escalation with high-dose 3D-conformal radiotherapy (HD-3D-CRT) or low-dose 3D-conformal radiotherapy plus HDR brachytherapy (LD-3D-CRT + HDR-B) for intermediate- or high-risk prostate cancer: higher PSA control with lower toxicity [abstract]. Urology. 2011;78(Suppl):S23.

13. Guix B, Bartina J, Tello J, Solé J, Quinzaños L, Lacorte T, et al. Treatment of intermediate- or high-risk prostate cancer by dose escalation with high-dose 3D-conformal radiotherapy (HD-3D-CRT) or low-dose 3D-conformal radiotherapy plus HDR brachytherapy (LD-3D-CRT + HDR-B) for: early results of a prospective comparative trial [abstract]. Int J Radiat Oncol Biol Phys. 2010;78 Suppl 1:S78.

14. Zwahlen DR, Andrianopoulos N, Matheson B, Duchesne GM, Millar JL. High-dose-rate brachytherapy with conformal external beam radiotherapy in the treatment of prostate cancer. Brachytherapy. 2010;9:27-35.

15. Sathya JR, Davis IR, Julian JA, Guo Q, Daya D, Dayes IS, et al. Randomized trial comparing iridium implant plus external-beam radiation therapy with external-beam radiation therapy alone in node-negative locally advanced cancer of the prostate. JCO. 2005;23:1192-9.

16. Kestin LL, Martinez AA, Stromberg JS, Edmundson GK, Gustafson GS, Brabbins DS, et al. Matched-pair analysis of conformal high-dose-rate brachytherapy boost versus external-beam radiation therapy alone for locally advanced prostate cancer. J Clin Oncol. 2000;18:2869-80.

17. Pieters BR, de Back DZ, Koning CC, Zwinderman AH. Comparison of three radiotherapy modalities on biochemical control and overall survival for the treatment of prostate cancer: a systematic reviev. Radiother Oncol. 2009;93:168-73. 
18. Edge SB, Byrd DR, Compton CC, Fritz AG, Greene FL, Trotti 3rd A, editors Cancer staging AJCC. 7th ed. New York: Springer; 2010.

19. D'Amico AV, Whittington R, Malkowicz SB, Schultz D, Blank K, Broderick GA et al. Biochemical outcome after radical prostatectomy, external beam radiation therapy, or interstitial radiation therapy for clinically localized prostate cancer. JAMA. 1998;280:969-74.

20. Cox JD, Stetz J, Pajak TF. Toxicity criteria of the Radiation Oncology Group (RTOG) and European Organization for Research and Treatment of Cancer (EORTC). Int J Radiat Oncol Biol Phys. 1995;31:1341-6.

21. Roach 3rd M, Hanks G, Thames Jr H, Schellhammer P, Shipley WU, Sokol GH, et al. Defining biochemical failure following radiotherapy with or without hormonal therapy in men with clinically localized prostate cancer: recommendations of the RTOG-ASTRO phoenix consensus conference. Int J Radiat Oncol Biol Phys. 2006;65:965-74.

22. Fijałkowski M, Białas B, Maciejewski B, Bystrzycka J, Ślusarek K. Three-dimensional (3d) real-time conformal brachytherapy - a novel solution for prosatae cancer treatment. Part II. A feasibility clinical pilot study. Nowotwory, journal of. Oncology. 2005:55:115-21.

23. Kal HB, Van Gellekom MPR. How low is the $\alpha / \beta$ ratio for prostate cancer? Int J Radiat Oncol Biol Phys. 2003;57:1116-21.

24. Nickers $P$, Hermesse J, Daneufbourg JM, Vanbelle S, Lartigau E. Which a/ß ratio and half-time of repair are useful for predicting outcomes in prostate cancer? Radiather Oncol. 2010;97:462-6.

25. Shaffer R, Pickles T, Lee R, Moiseenko V. Deriving prostate alpha-beta ratio using carefully matched groups, long follow-Up and the phoenix definition of biochemical failure. Int J Radiat Oncol Biol Phys. 2011;79:1029-36.

26. Nahum AE, Movsas B, Horwitz EM, Stobbe CC, Chapman JD. Incorporating clinical measurements of hypoxia into tumor local control modeling of prostate cancer: Implications for the a/b ratio. Int J Radiat Oncol Biol Phys. 2003:57:391-401.

27. Roberts SA, Miralbell R, Zubizarreta EH, Fowler JF, Hendry JH. A modelled comparison of prostate cancer control rates after high-dose-rate brachytherapy (3145 multicentre patients) combined with, or in contrast to, external-beam radiotherapy. Radiather Oncol. 2014;111:114-9.

28. Agoston P, Major T, Frohlich G, Szabó Z, Lövey J, Fodor J, et al. Moderate dose escalation with single-fraction high-dose rate brachytherapy boost for clinically localized intermediate- and high-risk prostate cancer: 5-year outcome of the first 100 consecutively treated patients. Brachytherapy 2011;10:376-84

29. Boladeras A, Santorsa L, Gutierrez C, Martinez E, Pera J, Pino F, et al. External beam radiotherapy plus single-fraction high dose rate brachytherapy in the treatment of locally advanced prostate cancer. Radiather Oncol. 2014;112:227-32

30. Dearnaley DP, Sydes MR, Graham JD, Aird EG, Bottomley D, Cowan RA, et al. Escalated-dose versus standard-dose conformal radiotherapy in prostate cancer: first results from the MRC RT01 randomised controlled trial. Lancet Oncology. 2007;8:475-87.

31. Zietman AL, Bae K, Slater JD, Shipley WU, Efstathiou JA, Coen JJ, et al. Randomized trial comparing conventional-dose with high-dose conformal radiation therapy in early stage adenocarcinoma of the prostate: long-term results from proton radiation oncology group/american college of radiology 95-09. J Clin Oncol. 2010;28:1106-11.

\section{Submit your next manuscript to BioMed Central and take full advantage of:}

- Convenient online submission

- Thorough peer review

- No space constraints or color figure charges

- Immediate publication on acceptance

- Inclusion in PubMed, CAS, Scopus and Google Scholar

- Research which is freely available for redistribution 